Mediterránea Ser. Biol. (1987), n. o 9. Pág. 5-13

\title{
ACTIVIDAD INVERNAL EN POBLACIONES DE LAGARTOS DE LA ZONA SUBTROPICAL
}

\author{
por \\ Jesús Mellado ${ }^{1}$ y Gaspar Olmedo ${ }^{1}$
}

\section{RESUMEN}

En la presente nota se describen los patrones de actividad invernal en poblaciones de lagartos para un gradiente que abarca doce grados de latitud $\left(26\right.$ a $\left.38^{\circ} \mathrm{N}\right)$ en el sur de Europa y Norte de Africa. Se usan para ello datos cualitativos reunidos en el curso de diez años de observaciones, que se complementan con otros de índole cuantitativa y mucho más rigurosos obtenidos en el curso de muestreos rutinarios a lo largo del ciclo anual de actividad. Los resultados obtenidos inducen a atribuir una gran importancia a la actividad en épocas desfavorables. Este fenómeno aparenta ser una característica propia del nivel de población y presenta una gran variabilidad en todos los niveles de organización considerados.

\section{WINTER ACTIVITY IN SUBTROPICAL LIZARD POPULATIONS}

\section{SUMARY}

This note describes general patterns of winter activity in lizard populations along a latitudinal gradient with an approximate range of twelve degrees $\left(26\right.$ to $\left.38^{\circ} \mathrm{N}\right)$ in southern Europe and North Africa. We make use of qualitative data gathered through the last ten years in the area, as well as much more accurate quantitative data obtained from periodic censuses covering the whole year cicle in certain populations. Results allow us to impute a great importance to the activity observed in the unfavourable season. This activity seems to be an attribute at the population level and shows a great variability between and within species and populations.

Es creencia tradicional y muy extendida el atribuir un período de latencia invernal estricta - salvo excepciones - a los reptiles que habitan en latitudes templadas (ÁNGEL, 1946; WITTE, 1948; SMITH, 1951; HELLMICH, 1962;

(1) Estación Experimental de Zonas Áridas. General Segura, 1. 04001. Almería. 
DOTTRENS, 1963; FRETEY, 1975; STREET, 1979, entre otros). Esta aseveración es sin duda cierta para amplias áreas de Europa Central y del Norte pero dista mucho de ser válido en zonas más meridionales. Así, mientras que en las primeras la generalidad de los reptiles entra en fase de letargo en fechas que suelen situarse en el mes de octubre para no volver a aparecer hasta bien entrado marzo (ver e.g. FRETEY, 1975; STREET, 1979, o NULAND y STRIJBOSCH, 1981), existen evidencias de que la actividad de los reptiles no se interrumpe durante los meses de invierno en latitudes más bajas (ver e.g. SAINTGIRONS, 1953; BONS, 1959; MELLADO et al., 1975; AMORES et al., 1980), aunque se desconoce la importancia relativa que esta actividad pueda tener respecto de la que se observa en épocas climáticas más favorables.

En la presente nota se trata de describir con algún detalle este fenómeno, aplicado al caso de las poblaciones de lagartos que aparecen en un rango que abarca doce grados de latitud (entre 26 y $38^{\circ} \mathrm{N}$ ) en el sur de Europa y norte de Africa y coincide «grosso modo» con la faja latitudinal definida como zona subtropical (ver LINCOLN, BOXSHALL y CLARK, 1982) en el área considerada. Se utilizan para ello abundantes evidencias cualitativas recogidas a lo largo de un período de diez años, principalmente en Andalucía pero también en el Sahara central argelino (enero de 1977), Islas Canarias (noviembre de 1977) y sur de Marruecos (región de Agadir, diciembre de 1984 y enero de 1985), en unión a datos que aparecen en la bibliografía. En épocas recientes se han completado estas observaciones con datos cuantitativos mucho más rigurosos, obtenidos mediante la realización de muestreos rutinarios realizados en poblaciones de lagartos del sureste de España, con una periodicidad de tres semanas a lo largo del ciclo diario. Este método supone una intensidad de muestreo uniforme a lo largo del ciclo estudiado, lo que posibilita la comparación directa de los resultados obtenidos en las diferentes épocas del año (MELLADO, 1985 y MELLADO y OLMEDO, 1985, desarrollan con detalle el método utilizado).

Se ha considerado aquí como invierno un trimestre cuyo centro coincide con el solsticio de invierno y que incluye pues los meses de noviembre, diciembre y enero, aproximadamente. Desde el punto de vista de la evolución de las variables climáticas, el solsticio supone la menor duración del fotoperíodo en el curso del año, y es en los meses de diciembre y enero cuando se dan los mínimos de radiación solar incidente y temperatura, respectivamente (ver por ejemplo OLMEDO et al., 1985), por lo que se considera más adecuado este «invierno» que el convencional, período que hay entre el solsticio de invierno y el equinocio de primavera, fecha en la que la actividad de los lagartos es ya generalizada en las regiones consideradas y cercana a los máximos del año en muchos casos (ver e.g. MELLADO, 1985).

A los efectos de este estudio, se considera que una población presenta actividad invernal cuando es común observar diferentes individuos de dicha población a lo largo del período que aquí se considera invierno, sin incluir pues observaciones excepcionales de individuos aislados, mucho más frecuentes y que serán valoradas posteriormente.

En la tabla I se da un resumen detallado de las especies de lagartos que presentan una actividad generalizada en el período invernal, con indicación del área geofráfica en que se observó dicho comportamiento. Se aprecia a primera 
GEKKONIDAE

Geckonia chazaliae

Quedenfeldtia trachyblepharus

Saurodactylus mauritanicus

Tarentola delalandii

Tarentola mauritanica

Tropiocoliotes tripolitanus

AGAMIDAE

Agama bibroni *

Agama mutabilis

CHAMAELEONIDAE

Chamaeleo chamaleon

SCINCIDAE

Chalcides mionecton

Chalcides sexlineatus

Chalcides viridanus

LACERTIDAE

Acanthodactylus boskianus

Acanthodactylus eythrurus

Acanthodactylus inornatus

Acanthodactylus pardalis

Gallotia atlantica

Gallotia galloti

Gallotia simonyi

Gallotia stehlinii

Mesalina guttulata

Mesalina olivieri

Podarcis hispanica

Podarcis sicula

Psammodromus algirus *

Psammodromus hispanicus
N Souss, Ma.

D Alto Atlas, Draa, Ma. (1)

N Souss, Ma.

$\mathrm{N}$ Islas Canarias

N General

N Draa, Ma. (2)

D Souss, Ma.

D Tademait, Ar.

D Andalucía (3)

O Souss, Ma.

O Islas Canarias

O Islas Canarias

D Tademait, Ar.

D Andalucía, Souss, Ma.

D Souss, Ma., Chardaïa, Ar.

D Souss, Ma.

D Islas Canarias

D Islas Canarias

D Islas Canarias

D Islas Canarias

D Ghardaïa, Ar.

D Souss, Ma.

D General

D Almería

D Andalucía

D Andalucía

Observaciones de individuos aislados en: Ptyodactylus hasselquistii (N; Tademait, Ar.); Uromastix acanthinurus (D; Tarhit, Ar.); Chalcides bedriagi $(\mathrm{O}$; Andalucia); Chalcides ocellatus (O; Souss, Ma.); Trogonophis wiegmani (O; Souss, Ma.)

Tabla I: Lista de especies de lagartos que presentan actividad a nivel de población en el área considerada. N: especies nocturnas; D: diurnas; O: ocultas (ver detalles en el texto). Ma.: Marruecos; Ar.: Argelia; el resto de las observaciones en territorio español.

1) Según SAINT-GIRONS (1953) y BONS (1959); 2) según BONS (op.cit.); 3) Según SANTO-ROSA et Al. (1985).

* Actividad en individuos juveniles, principalmente.

vista que la actividad invernal a nivel de población es un fenómeno muy extendido que afecta a numerosas especies de lagartos en el rango de latitudes considerado, donde se incluyen regímenes climáticos muy diferentes que van del subtropical húmedo de las Islas Canarias hasta el desierto extremo de Argelia central, pasando por los diferentes tipos de clima mediterráneo. 


\begin{tabular}{|c|c|c|c|c|}
\hline Especie & Localidad & Sexo/edad & $\mathrm{N} / \mathrm{N} \max$. & $\%$ \\
\hline \multirow[t]{3}{*}{ P. hispanicus } & $\begin{array}{l}\text { Cueva de los Medina } \\
\text { (Esparto) }\end{array}$ & ads. & $3 / 10$ & 30.0 \\
\hline & Cueva de los Medina & oro & $3 / 14$ & 21.4 \\
\hline & (Tomillar) & & & \\
\hline \multirow[t]{6}{*}{ A. erythrurus } & Rambla Morales, S & juvs. & $1 / 4$ & 25.0 \\
\hline & Rambla Morales, A & juvs. & $1 / 7$ & 14.3 \\
\hline & Alicante (1) & juvs. & $2 / 72$ & 2.8 \\
\hline & & \$ & $1 / 29$ & 3.4 \\
\hline & Cádiz (2) & juvs. & $28 / 118$ & 23.7 \\
\hline & & ads. & $3 / 103$ & 2.9 \\
\hline$P$. algirus & Alicante (1) & & $4 / 39$ & 10.3 \\
\hline
\end{tabular}

Tabla II: Importancia relativa de la actividad invernal en diferentes poblaciones de lagartos epígeos diurnos del sur de España. Se representa el número de individuos observados en un censo diario ( $\mathrm{N}$ ) respecto del número máximo ( $\mathrm{N}$ max.) observado en el curso del ciclo anual completo para dicho intervalo. Basado en datos que aparecen en MELLADO (1985) excepto en los casos de Alicante y Cádiz que figuran en (1) SEVA (1982) y (2) BUSACK (1976), respectivamente.

Fecha/sexo,

\begin{tabular}{|c|c|c|c|c|c|c|}
\hline edad & $\begin{array}{l}\mathrm{N} / \mathrm{N} \\
\mathrm{max} .\end{array}$ & $\%$ & $\begin{array}{l}\mathrm{N} / \mathrm{N} \\
\max .\end{array}$ & $\%$ & $\begin{array}{l}\mathrm{N} / \mathrm{N} \\
\max .\end{array}$ & ం'ం' \\
\hline 051182 & $32 / 32$ & 100 & $9 / 21$ & 42.8 & $10 / 30$ & 33.3 \\
\hline 2911 & 3 & 9.4 & 1 & 4.8 & 4 & 13.3 \\
\hline 1512 & 9 & 28.1 & 0 & - & 1 & 3.3 \\
\hline 040183 & 4 & 12.5 & 0 & - & 2 & 6.7 \\
\hline 2501 & 6 & 18.7 & 6 & 28.6 & 9 & 30.0 \\
\hline
\end{tabular}

Tabla III:Importancia relativa de la actividad invernal de Podarcis sicula en el Parque de Almería. Leyenda como en la figura anterior.

Este fenómeno afecta a una parte considerable del conjunto de la fauna de lagartos existentes en cada región. Así, al considerar las tierras bajas de Andalucía, siete de las once especies $(63,6 \%)$ que aparecen en el área prolongan su actividad durante el invierno (no se observan Chalcides chalcides, Ch. bedriagai, Lacerta lepida ni Blanus cinereus), porcentaje similar al que se observa en el Oued Massa (región de Agadir) donde la actividad invernal generalizada incluye a nueve de las quince especies existentes $(60 \%$; no se observa en 
Chamaeleo chamaleon, Chalcides ocellatus, Eumeces schneideri, Sphenops sphenopsiformis, Acanthodactylus boskianus ni en Trogonophis wiegmanni) e inferior al que se aprecia en las Islas Canarias donde la práctica totalidad de las especies existentes continúa su actividad en invierno».

Los resultados anteriores dan una idea general de la amplitud del fenómeno en el conjunto de la fauna paro no de la importancia relativa de esta actividad en el seno de las diferentes poblaciones consideradas. Una manera de valorar dicha importancia consiste en comparar los niveles de actividad detectados en la época invernal con los encontrados en aquellas épocas donde la actividad es máxima. En las tablas II y III se resumen resultados de este estilo para aquellas poblaciones donde se poseen datos cuantitativos para el conjunto del ciclo anual.

Se aprecia a primera vista que la importancia relativa de la actividad invernal presenta una gran variabilidad según la especie, población y clase de sexo y edad dentro de ésta. Así, se pueden encontrar niveles apreciables - por ejemplo, mayores del veinte por ciento- en siete de los trece $(53,8 \%)$ ejemplos considerados, que pueden ser muy importantes en el caso de las hembras de $P$. sicula o en los juveniles de esta misma especie, que presentan su máximo anual a principios de noviembre, aunque existan otros en que la actividad invernal es muy reducida en relación con el máximo anual.

Se puede observar también que dicha actividad fluctúa considerablemente en el curso del invierno para un mismo item. Estos cambios pueden ser muy importantes, como se observa en las diferentes clases de sexo y edad en $P$. sicula (tabla III), donde los juveniles pueden oscilar entre el máximo de actividad y sólo un diez por ciento de ésta en el curso del mismo invierno. Estos cambios pueden desarrollarse en lapsos muy cortos de tiempo en situaciones de clima variable; en la figura 1 de ilustra un ejemplo para el agregado de $A$ cant-

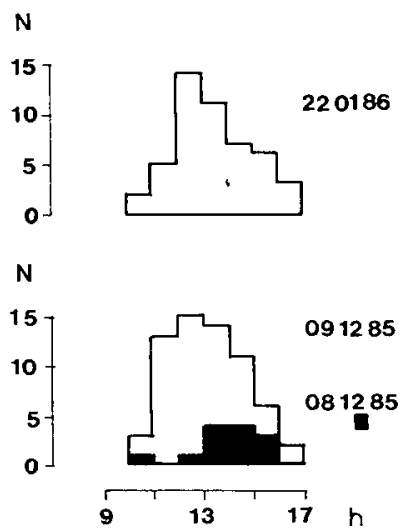

Figura 1.-Ritmos diarios de actividad en Acanthodactylus del Oued Massa. Se representa el número de observaciones $(\mathrm{N})$ realizadas en censos horarios para tres días completos del invierno 1985-1986. El área oscura representa el nivel de actividad en condiciones climáticas muy desfavorables (bruma y vientos fuertes del norte). Nota: no se ha hecho distinción entre las tres especies ( $A$. erythrurus, $A$. inornatus y $A$. pardalis) que aparecían en el transecto. 


\section{P.sicula}

ơ

N

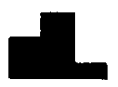

ㅇํ

juv.

fecha

等

${ }^{5}$
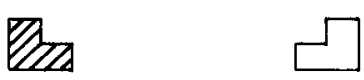

250183

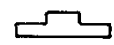

040183

$0^{5}$

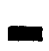

[2]

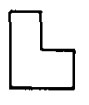

151282

$0^{5}$
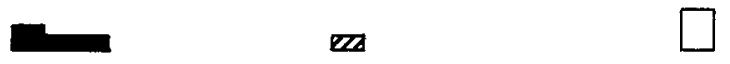

291182
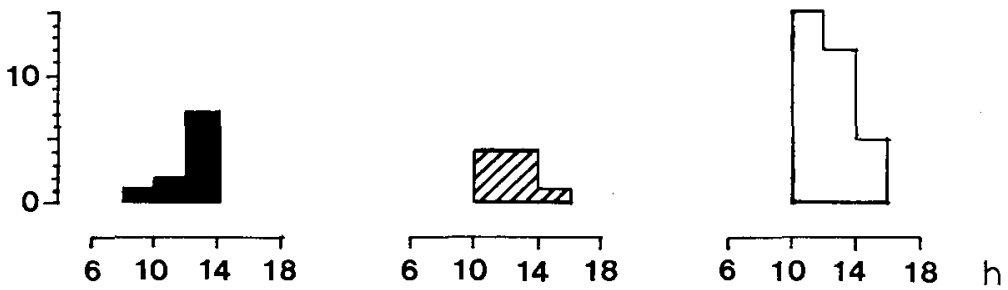

051182

Figura 2.-Ritmos diarios de actividad en $P$. sicula. Se representa el número de individuos (N) observados a lo largo de un ciclo diario completo para machos, hembras y juveniles, así como la fecha en que se realizó cada censo.

hodactylus del Oued Massa, donde la actividad registrada un día de viento con claros y nubes alternados fue cinco veces $(20 \%)$ menor que la observada el día siguiente, soleado y sin viento.

Se ha visto en lo anterior que el mantenimiento de la actividad en las épocas más desfavorables del año es lugar común en las poblaciones de lagartos de las latitudes consideradas. Una característica fundamental de este fenóme- 
no es la gran variabilidad que muestra la importancia relativa de dicha actividad en todos los niveles considerados, entre especies, poblaciones, clases de sexo y edad y aún dentro de éstas, entre diferentes fechas. Sin embargo, esta variabilidad no parece aleatoria sino que puede ponerse en clara relación con la evolución de determinadas variables climáticas en la época considerada. Así, OLMEDO et al., (1985) detectan en invierno una dependencia significativa del nivel de actividad de adultos y juveniles de $P$. sicula con la radiación incidente y con la temperatura máxima, respectivamente, y en general, los cambios que se observan a lo largo del ciclo anual corresponden a unas tendencias cíclicas en el ritmo anual de actividad, claramente definidas (ver MELLADO, 1985). En la figura 2 se ilustran estas tendencias para $P$. sicula en invierno, época que suele corresponderse con mínimos en el número de observaciones (machos y juveniles; ver otros ejemplos en la tabla II) o bien supresión de la actividad por períodos de tiempo, generalmente cortos, de clima desfavorable (hembras de P. sicula)

Los resultados obtenidos en este estudio permiten atribuir mínimos relativos a la actividad que desarrollan los lagartos en la época invernal y en las latitudes señaladas, pero existen indicios que apuntan, como ya señalara BONS (1959), a que ésta sea la época de máxima actividad en algunas situaciones. Así, la mayor parte de las hembras de $A$. boskianus observadas en el plateau de Tademait (Argelia) presentaban huevos oviducales muy desarrollados a finales de enero, lo que supone una actividad invernal equivalente a la que se observa en primavera y verano en otras poblaciones. Del mismo modo, el máximo de actividad del agregado de Acanthodactylus de Massa se observa en diciembre, con una densidad aparente de 132 individuos por hectárea frente a los 115 que se registran en julio, época en que se da un máximo secundario (MELLADO, manuscrito inédito).

La variabilidad que se observa en el nivel de actividad invernal entre y dentro de las poblaciones estudiadas puede explicar la que se detecta dentro de las especies y en el conjunto de la fauna. Al nivel de especies, aunque es posible encontrar ejemplos en que se observa una actividad invernal muy importante en numerosas poblaciones dentro de su área de distribución, caso de Podarcis hispanica en las cordilleras del centro de España (PÉREZ MELLADO, 1981); Sierra Morena (MELLADO et al., 1975), Sevilla, Granada, sureste de Almería, Rif (Xauen) o Atlas de Blida (cercanías de Argel), lo más frecuente es que dicha actividad se observe en determinadas poblaciones pero no en otras. Así, C. chamaleon permanece activo en la provincia de Cádiz (ver SANTO-ROSA et al., 1985) pero no en el Oued Massa, área donde es muy común en verano (Abdelaziz ALLABOU, com. pers.) pero no se observa en la época invernal. $A$. boskianus por su parte no aparece en invierno en Massa pero sí en Tademait (ver más arriba), del mismo modo que $U$. acanthinurus experimenta una interrupción de su actividad en el Sahara central (Tahrit) pero no en la región de Zemmur (HEIM DE BALZAC, citado en VALVERDE, 1957) y en conclusión, el mantenimiento de la actividad invernal parece ser un atributo más propio de las poblaciones concretas que de las especies como un todo.

Lo mismo se puede decir para el conjunto de la fauna. Como se aprecia en la tabla I, la actividad invernal es un fenómeno que se puede observar en 
numerosas especies que no sólo están muy diferenciadas filogenéticamente, sino que presentan también diferentes formas de vida que afectan al modo de captación de energía externa, recurso limitante en época climáticamente desfavorables (ver BOGERT, 1949; SAINT-GIRONS, 1956; SAINT-GIRONS, 1971). En efecto, es posible encontrar dicha actividad tanto en especies diurnas (D) que termoregulan básicamente por heliotermia, como en especies ocultas (O: excavadoras, lapidicolas, etc.; MELLADO, 1985, da una detallada descripción de estos grupos funcionales) y nocturnas $(\mathrm{N})$ donde la tigmotermia juega un papel fundamental en la regulación de su temperatura.

$\mathrm{El}$ hecho de que no se observen diferencias significativas al valorar las proporciones de los diferentes grupos funcionales en invierno frente a las que se observan en la época de máxima actividad (0.68:0.12:0.2, versus $0.75: 0.17: 0.07$; $\mathrm{G}=0.68 ; 2 \mathrm{~g} .1$. Los datos para la última época en MELLADO, 1985), sugiere que dicha actividad es independiente del tipo de vida de las diferentes especies.

Sin embargo, este fenómeno no es independiente del tamaño. Al clasificar las diferentes especies en grandes (por ejemplo, mayores de $100 \mathrm{~mm}$. de LCC. POUGH, 1980, describe las relaciones longitud-masa y la importancia de este último parámetro en los intercambios de energía en ectotermos) y pequeñas, se observa que la proporción de especies grandes que presentan reposo invernal es mucho mayor que la de pequeñas $(61.6 \%$ frente a $17.4 \%)$ y esta diferencia es estadísticamente significativa $(\mathrm{G}=7.252,1 \mathrm{~g} .1 ., \mathrm{P}<0.01)$

\section{AGRADECIMIENTOS}

Vaya nuestro más sincero agradecimiento a la colaboración prestada en el campo por Miguel Arbella - infatigable buscador de Agamas- Lina Rodríguez, Teodoro Marañón, Abdelaziz Allabou y Boubker Alaoui. A Miguel Ulpiano y a Melitón Cárdenas, por las facilidades prestadas en el sur de Marruecos. Las expediciones a Árgelia y Marruecos fueron financiadas parcialmente mediante una bolsa de viaje y una misión de asistencia técnica del M. ${ }^{\circ}$ de Asuntos Exteriores de España, al primer autor. 


\section{BIBLIOGRAFÍA}

ÁNGEL, F. (1946). Reptiles et Amphibiens. Faune de France, 45. Lechevalier, París.

AMORES, F., A. FRANCO, F. HIRALDO y J. MELLADO (1980). Actividad invernal de reptiles en el S. W. español. II Reunión Iberoamericana de Conservación y Zoología de Vertebrados, Cáceres (resumen).

BOGERT, C. M. (1949). Thermoregulation in reptiles, a factor in evolution. Evolution, 3: 195-211.

BONS, J. (1959). Les lacertiliens du sud-ouest marocain. Trav. Inst. Scient. Cherif. ser. Zool. 18.

BUSACK, S. D. (1976). Activity cycles and body temperatures of Acanthodactylus erythrurus. Copeia, 4: 826-830.

DOTTRENS, E. (1963). Batraciens et reptiles d'Europe. Delachaux et Nestlé, Neuchâtel, Suisse.

FRETEY, J. (1975). Guide des Reptiles et Amphibiens de France. Hatier, París.

HELLMICH, W. (1962). Reptiles and Amphibians of Europe. Blandford. London.

LINCOLN, R. J., G. A. BOXSHALL y P. F. CLARK (1982). A dictionary of ecology, evolution and systematics. Cambridge University Press, Cambridge.

MELLADO, J. (1985). Agregados de lagartos mediterráneos en el espacio y en el tiempo. Tesis Doctoral, Universidad de Sevilla, Sevilla.

MELLADO, J., F. AMORES, F. F. PARREÑO y F. HIRALDO (1975). The structure of a mediterranean lizard community. Doñana, Acta Vert., 2: 145-160.

MELLADO, J. y G. OLMEDO (1985). Un método de análisis de ciclos de actividad en lagartos. En: Avances sobre investigación en bioclimatología. (A. BLANCO DE PABLOS, ed.) pp. 291-310. CSIC, Salamanca.

NULAND, G. J. van, y H. STRIJBOSCH (1981). Annual rhythmics of Lacerta vivipara and Lacerta agilis agilis. Amphibia-Reptilia, 2: 83-95.

OLMEDO, G., R. LÁZARO y J. MELLADO (1985). El clima y su relación con el ciclo de actividad de Podarcis sicula. En: Avances sobre la investigación en Bioclimatología (A. BLANCO DE PABLOS, ed.) pp. 311-330. CSIC, Salamanca.

PASTEUR, G (1981). A survey of the species groups of the Old World scincid genus Chalcides. Journal of Herpetology, 15: 1-16.

PÉREZ MELLADO, V. (1981). Los lacertidae del oeste del sistema central. Tesis Doctoral, Universidad de Salamanca, Salamanca.

POUGH, F. H. (1980). The advantages of ectothermy for tetrapods. Am. Nat., 115: 92-112.

SAINT-GIRONS, H. (1953). Note sur les périodes de latence des reptiles au Maroc. Bull. Soc. Zool. France, 78: 377-381.

- (1971). Quelques facteurs du rythme circadien d'activité chez les lépidosauriens (reptiles). Bull. Soc. Zool. France, 96: 317-330.

SAINT-GIRONS, H. y M. C. SAINT-GIRONS (1956). Cycle d'activité et thermorégulation chez les reptiles (lézards et serpents). Vie et Milieu, 7: 133-226.

SANTO-ROSA R., M. RODRÍGUEZ, J. C. RUBIO GARCÍA y J. C. NEVA (1985). Actividad invernal del camaleón común (Chamaeleo chamaleon). en: Avances sobre la investigación en bioclimatología (A. BLANCO DE PABLO, ed.) pp. 341-351. CSIC, Salamanca.

SEVA, E. (1982). Taxocenosis de Lacertidos de un arenal costero alicantino. Secretariado de publicaciones, Universidad de Alicante, Alicante.

SMITH, M. (1951). The British Amphibians and Reptiles. Collins, London.

STREET, D. (1979). The reptiles of northern and central Europe. Batsford, London.

VALVERDE, J. A. (1957). Aves del Sahara español. (Estudio ecológico del desierto). Inst. Est. Afri., Madrid..

WITTE, G. F. de. (1948). Amphibiens et reptiles. Faune de Belgique. Musée Royal d'Histoire Naturelle, Bruxelles. 